\title{
Data Delivery Increment using CVAR Protocol for Urban VANET
}

\author{
M. Manoj, M.E \\ Dept. of Electronics and Communications Engineeering \\ Easwari Elngineering College, Anna University, \\ Chennai, India
}

\begin{abstract}
In Vehicular Ad Hoc Network due to high dynamic the data dissemination makes a big issue. Delivery ratio getting low in the road junctions as the vehicles departure into different directions. Existing schemes such as Mobile cluster assisted routing for urban VANET has attempted to increase the delivery ratio in the junction area. But in a real time scenario, the vehicular density is very high at the junction region compared with highways. Our proposed system improves the data delivery ratio at the junction region even when the high vehicular density. This paper introduces a routing protocol called Cluster Vehicular Assisted Routing (CVAR) for VANET. The main motto of the proposal is to increase the performance of VANET in the junction region. To increase the data dissemination at the junction, the network is effectively spare into many clusters and eager to move along with the vehicles towards the destination. The moving nodes and cluster formation at junction are considerably improves the connection time and the delivery ratio.
\end{abstract}

\section{Keywords}

VANET, Vehicular density, Mobility, Cluster

\section{INTRODUCTION}

A vehicular Ad hoc Network or VANET is a special case in Mobile Ad hoc network, to provide communications among close by vehicles and between vehicles and nearby fixed equipment, usually described as roadside equipment. The main goal of VANET is providing safety and comfort for passengers. To this end a special electronic device will be placed inside each vehicle which will provide Ad hoc Network connectivity for the passengers. This network tends to operate without any infra-structure or legacy client and server communication. Each vehicle equipped with VANET device will be a node in the Ad hoc network and can receive and relay others messages through the wireless network.

Most of the concerns of interest to MANETs are of interest in VANETs, but the details differ. Rather than moving at random, vehicles tend to move in an organized fashion. The interactions with roadside equipment can likewise be characterized fairly accurately. And finally, most vehicles are restricted in their range of motion, for example by being constrained to follow paved highway. High mobility leads to frequent broken routes in VANET.

Moreover, the vehicular network brings additional problems related to the road junction regions. For lower vehicular density, a sender sends the packets at the intersection of the roads may not be able to find the connecting path to the receiver who may be out of coverage region. This problem was attempted to solve by using static-node assisted adaptive data dissemination protocol for vehicular networks (SADV) [1], which enables packets to be buffered at the intersection until the connecting path is available. When considering the urban areas, the vehicular density is always high and attains it peak during the heavy traffic hours. A single static node placed at the junction is not expected to store such a large volume of data and to disburse in different directions within the connection time possible.

This paper has makes an effort to increase the total number of packets transfer and increment in bandwidth of static node placed in the junction. A novel routing mechanism cluster vehicular assisted routing protocol (CVAR) assisted by the vehicular clusters is proposed here to offset mainly the problems arising out of high vehicular density. It is designed with the existing traffic infrastructures to cluster the network effectively for a better delivery ratio and to reduce broadcast storms. Nodes at the junction are organized as a set of clusters in which one node called cluster head collects data from the static node and sends them to the actual destination. Cluster assisted routing will aim to provide less propagation delay and high delivery ratio with bandwidth fairness too. Combination of cluster assisted dissemination mechanism with the static node approach is able to tackle the high vehicular density effectively. Rest of the paper is organized as follows: Section II Presents the related works. Section III details the protocol design and performance evaluation, simulation results are brought out in Section IV. Finally, Section V concludes this paper.

\section{RELATED WORK}

The related works reported in vehicular ad- hoc routing are brought out in this section to study the impact of the models and schemes incorporated within. Ai Huo Ho[5] proposed a CB-S broadcast technique for the street environment. The CB-S can always successfully disseminate the message to every node in the street network. This perfect coverage is achieved with the least overhead. The CB-S can always disseminate messages to all the nodes in the least amount of time using the least number of rebroadcast nodes.

YuhaoWangy et al. [2] in their work on Performance Evaluation of Routing Protocol in VANET with Vehicular Density analyzed the properties of the two mobility models in high density urban areas and all simulations results shows the distinction between reactive and proactive routing protocol. It indeed, informs drivers and other passengers of potentially dangerous traffic situations while there is still time to avoid them.

Rodolfo Oliveira et al. [7] presented a new method that uses the available knowledge about the network's topology to improve the routing protocol's performance through decreasing the probability of path breaks that integrated new improvements in the OLSR routing protocol. 
In this section, existing schemes on data dissemination, vehicular routing and vehicular density are dealt with. Some of the methods such as OLSR [9] only perform well in high vehicular density. Data disseminate, when the direction of connectivity changes at the road junctions, becomes a major issue in the routing of the vehicular communication. Vehicle-toinfrastructure (V2I) communication has shown some advantages compared to the vehicle-to-vehicle (V2V) communication such as supporting road junctions, communication protocols and vehicular density.

\section{PROPOSED MODEL}

The CVAR is proposed protocol and it considers the roadmap of an area, which mainly contains junctions with high vehicular density. Some of the positioning services are used in each node is aware of its location, such as Global positioning system. This enables the position of the node, direction prediction and its velocity. The protocol operates in two ways namely, high vehicular density area and low vehicular density area.

\subsection{High Vehicular Density}

As the vehicle approaches a road junction, it works on high vehicular density. The static node gets immediate environment and detects clusters. The cluster in the direction of the destination is chosen to forward the packet. One of the vehicles within the chosen cluster is elected as the cluster head which gather the data from various nodes. Formation of the clusters in junction mode is shown in Fig.1.

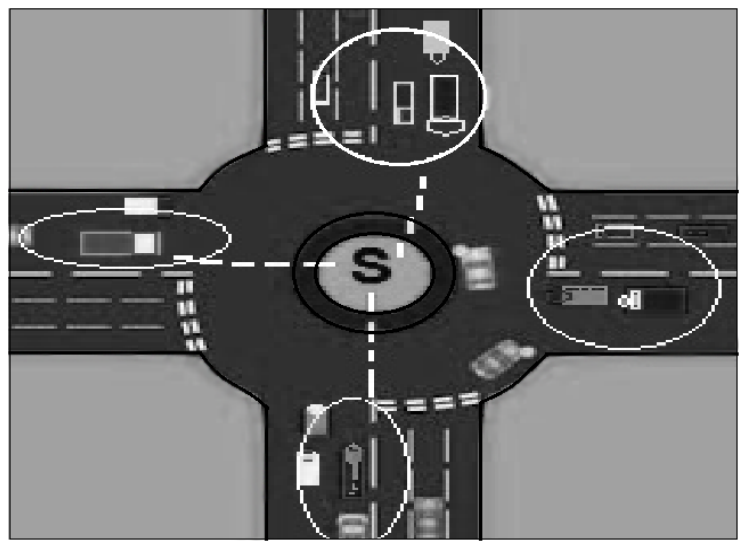

Fig. 1 Creation of vehicle clusters at the junction

\subsection{Low Vehicular Density}

At regular interval of time, each vehicle node receives the signal from the static node. If it comes within the communication region of the static node, the vehicle node stays in high vehicular density otherwise it is works for low vehicular density. In highway mode, vehicles use vehicle to vehicle communication forwarding and also deliver data to the actual destination. A mobile cluster carrying a cloud of vehicles is shown in Fig.2. This situation stay connects till the mobile cluster reaches the next junction.

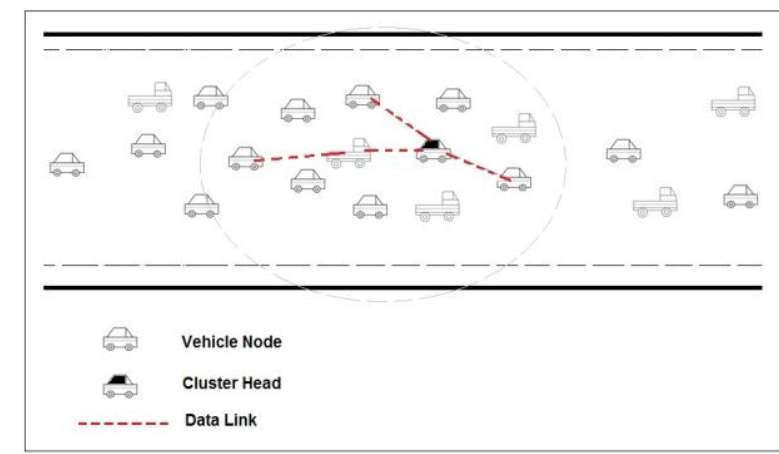

Fig. 2 Data delivery from the cluster head

\subsection{Formation of Cluster}

Let us consider the stage at junction, which contains $\mathrm{N}$ no of vehicles and a static node. The static node gives a high bandwidth data communication to the vehicles. Consider the vehicle node $\mathrm{A}$ having the velocity of $\mathrm{Va}$, a direction vector $\mathrm{dx}$,dy vectors are Cartesian coordinates (Xa, Ya). Now consider the static node as a center point having Cartesian co-ordinates (Xs, Ys).

Step 1: The directional vector of vehicle A towards static node $\mathrm{S}$ is given by:

$$
\overrightarrow{\mathrm{AS}}=\left(\mathrm{x}_{\mathrm{A}}-\mathrm{x}_{\mathrm{S}}, \mathrm{y}_{\mathrm{A}}-\mathrm{y}_{\mathrm{S}}\right)
$$

Step 2: The velocity vector of vehicle $A$ is calculated as

$$
\overrightarrow{\mathrm{V}_{\mathrm{a}}}=\overrightarrow{\mathrm{dx}_{\mathrm{a}}} \cdot \mathrm{v}_{\mathrm{a}}+\overrightarrow{\mathrm{dy}} \cdot \mathrm{v}_{\mathrm{a}}
$$

Step 3: Distance Vector has the directional similarity value, which is the dot product of direction vector and velocity vector

Distance Vector $=\overrightarrow{\mathrm{V}_{\mathrm{a}}} \cdot \overrightarrow{\mathrm{SA}}$

The Distance Vector of the vehicle gets negative value which travels in the opposite direction will be omitted by the cluster.

\subsection{Announcement of Cluster Head}

Each moving cluster has its own cluster ID and it Covers several vehicles. By using Algorithm-1, cluster head is chosen for mobile cluster where a count on vehicle nodes and static node becomes the input and cluster head is the output. The purpose of the algorithm is to select a cluster head which having the maximum coverage. Once the cluster head is being selected, the distance between the static node and the cluster head is being calculated as:

Distance $=\operatorname{sqrt}(($ xa-xs $) 2+($ ya-ys $) 2)$

\section{Algorithm-1}

Input : Na, $\{\eta(\mathrm{nx}), \xi(\mathrm{ny}), \mathrm{ti}(\mathrm{nz})\} \forall$ ny $\in \mathrm{Na}$

Output : R(na)

$\mathrm{R}(\mathrm{na}) \Leftarrow \phi$

coverage $\Leftarrow 0$

for each_neighbor $\mathrm{nx} \in \mathrm{Na}$ do

if $\eta(\mathrm{ny}) \geq$ kest and ny is $\mathrm{CH}$ then

$\mathrm{R}(\mathrm{na}) \Leftarrow \mathrm{R}(\mathrm{na}) \cup \mathrm{nz}$

update (coverage)

remove_from_list(ny, $\mathrm{Na}$ ) 
end if

Nord $=$ sort by descendent $(\mathrm{Na})$

while coverage $<100 \%$ do

remove_from_list(ny, Nord)

$\mathrm{R}(\mathrm{na}) \Leftarrow \mathrm{R}(\mathrm{na}) \cup \mathrm{nz}$

update (coverage)

end

\subsection{Vehicular Cluster In Highway Mode}

Once the traffic signal is turned on, each cluster starts moving in its destined direction. When the cluster moves away from coverage range of the junction region, cluster head starts announcing its status to all vehicles present within its cluster. The Mobile cluster incorporates a multi-hop relaying technique to forward the packets to the actual destination. When compared to flooding, it reduces the number of redundant packets in the network. Cluster head selects a set of relay nodes $\mathrm{R}$ within one hop region from its location using Algorithm-2 provided they are moving in the same direction along with $\mathrm{CH}$. In case the number of one-hop relay nodes are inadequate to cover the range, two-hop neighbors are also added to $\mathrm{R}$. By using these relay nodes $\mathrm{CH}$ disseminates the packets to the destination.

Protocol is summarized as follows. One-hop neighbors na that have stable links with Cluster Head are added one by one to R. This is the same rationale adopted in Algorithm-1. Because these links are stable, it indicates that they have higher probability of remaining active. Initially, the input variable OLD R assumes to be an empty vector. If the number of relay nodes selected within the one-hop range is not guaranteeing full coverage, the algorithm selects more relay nodes from two-hop count to replace some of the neighbor nodes na who have the lowest stability value. In case of tie with the stability values, the algorithm prefers $\mathrm{R}$ that covers nodes at two-hops away for a faster coverage. The additional Rs are stored in the vector OLD R.

The steps given in the following pseudo code summarizes the overall process flow of the proposed routing protocol. It also captures the data movement from static node to actual destination. The switching between junction and highway modes is clearly indicated in the flow. Mobile cluster, having formed with its relay nodes, takes care to deliver the packets to the destination before it reaches the next junction. If the destination changes its direction before completing the delivery of packets, the relay node will deliver to the static node and it switches to junction mode.

\section{Pseudo code for cluster vehicular assisted routing:}

If junction (High vehicular Density)

If fixed node assisted

get node direction \& angle velocity from the cluster using arbitral vector

\section{Choose cluster head}

Fixed node sends packets to cluster head

end if

else

Switch to road (Low vehicular Density)
Cluster head announces its cluster ID

Select 1-hop \& 2-hop neighbors as R

Cluster head sends packets to $\mathrm{R}$ nodes

$\mathrm{R}$ nodes deliver the packets to destination

end if

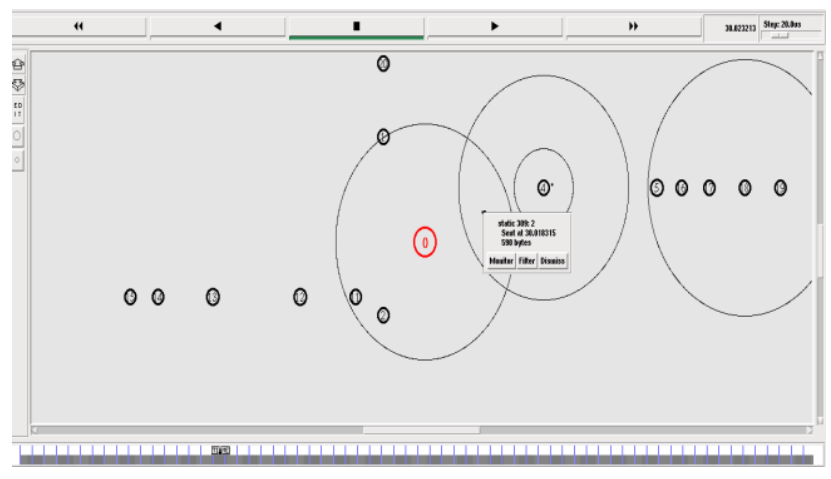

Fig. 3 Movement of data at the junction

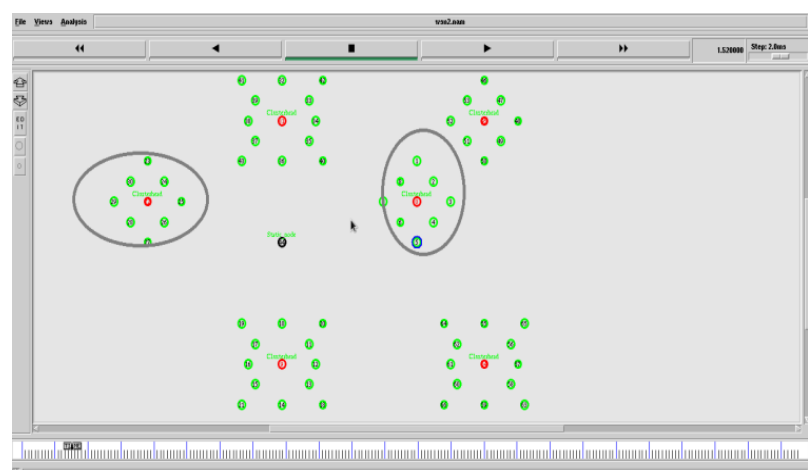

Fig. 4 Vehicular clusters moving away from junction

\section{SIMULATION AND PERFORMANCE EVALUATION}

\subsection{Mobility Model}

Mobile cluster formed on the low vehicle density mode is given in Fig. 4 after the traffic signal turns green. Clouds of vehicle nodes moving have been shown in fig 4 and these clouds continue till they reach their next respective intersections. These mobile clusters tend to increase connection time the nodes so the packets would be successful.

Generally simulation requires a mobility model. In order to observe vehicle movements in the metropolitan scenario the mobility model is preferred, which contains a grid road topology. The default parameters when the vehicles reach intersections are: probability of continuing straight is 0.5 and the probabilities of both turning right and turning left is 0.25 . The duration of red and green signal are set to $15 \mathrm{~s}$ and $10 \mathrm{~s}$ respectively.

\subsection{Simulation}

The traffic simulation tool, MobiSim [6] and the network simulator, NS2 version 2.35 [8] are utilized to simulate the proposed protocol. The simulation parameters that are configured for the experiment are summarized in Table I. 
TABLE I

\begin{tabular}{|c|c|}
\hline Parameter & Value \\
\hline Simulation area & $2000 \mathrm{~m} \times 2000 \mathrm{~m}$ \\
\hline Number of intersections & 4 \\
\hline Distance b/w Intersections & $500 \mathrm{~m}$ \\
\hline Number of vehicles & 35 to 85 \\
\hline Speed of vehicles & 20 to $60 \mathrm{mps}$ \\
\hline MAC protocol & IEEE $802.11 \mathrm{p}$ \\
\hline Data rate & $2 \mathrm{Mbps}$ \\
\hline Packet size & 512 bytes \\
\hline
\end{tabular}

\subsection{Simulation Results and analysis}

Samples of the screen shots while simulating the protoco CVAR are shown in the Fig.3 and Fig.4. Data transfer from vehicle to static node at the road junction is illustrated in Fig.3. The situation depicts the junction mode operation as described in Section IIIA. Static node, when the traffic signal is turned on to red, it receives the data from the nodes at the junction.

To analyze the protocol comprehensively, CVAR is compared with other two typical routing protocols namely SADV and GPSR. In this paper, in order to evaluate the protocol's flexibility to node density, the number of nodes is varied from 30 to 80 . Similarly, to evaluate the protocol's adaptability to node speed, the node speed is varied from $20 \mathrm{mps}$ to $60 \mathrm{mps}$. The simulation results are shown in Fig 5 and Fig 6. It is observed from Fig 3 that CVAR and SADV have performed well to display a high delivery ratio at lower node density. GPSR fails to deliver the packets as this does not consider the junction characteristics. When nodes in the network are sparse, say between 30 and 40, CVAR performs better with as much as $22 \%$ increase compared to GPSR and as much as $16 \%$ increase compared to SADV. When node density is comparatively high say, from 50 to 80 , the delivery ratio of CVAR remains high. Moreover, CVAR performs well in terms of scalability than other ones and its delivery ratio reaches up to $80 \%$.

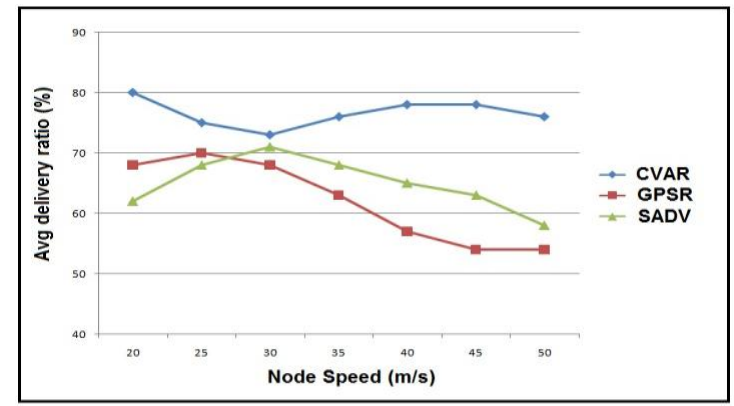

Fig.5. Average delivery ratio for node speed $30 \mathrm{mps}$

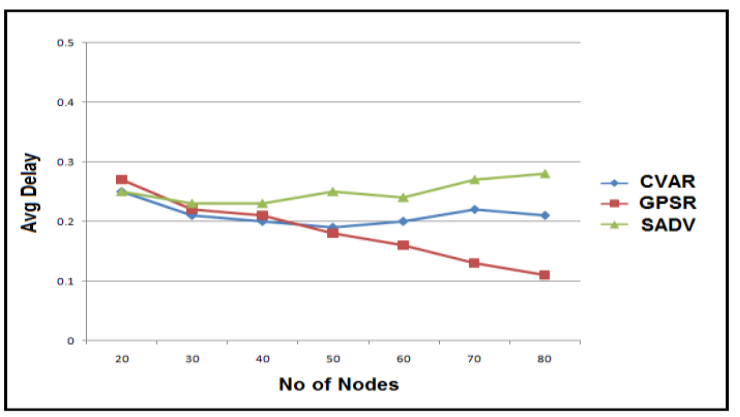

Fig.6. Average end-to-end delay for node speed is $\mathbf{3 0}$ mps
Fig 6 shows the average end-to-end delay of the protocols versus node density. It is observed that GPSR and CVAR exhibit a moderate variation in the delay while increasing the number of nodes. The degrading performance of SADV compared to CVAR in the case of average end-to-end delay is due its dependence on node density and speed. CVAR handles a high packet delivery as the node density and speed increases and the delay shoots up. Mobile cluster, most of the time helps to stay connected for a longer period at least till the next road intersection.

\section{CONCLUSION}

This paper proposed a new technique CVAR, a routing protocol for vehicular networks, which improves the performance of VANET even if the vehicular density is high. Static node mechanism combined with the features of moving clusters has been taken advantage of enhancing the packet delivery ratio and delay. It is evaluated the performance of CVAR in an urban roadmap with more cross roads. Under MobiSim and NS2 simulators, CVAR performance in terms of node density and speed is compared with two other routing protocols. It is observed from the analysis of the simulation results that CVAR routing mechanism is able to adapt to scalability and frequent topological change by extending the connection lifetime of mobile nodes using moving clusters so as to improve the performance of VANET. It can adapt to VANET in urban scenarios and mainly in junction regions commendably.

\section{REFERENCES}

[1] Yong Ding and Li Xiao, SADV: "Static-Node-Assisted Adaptive Data Dissemination in Vehicular Networks," IEEE Trans.Veh.Technol., vol. 59, no.5, June. 2010.

[2] Jing Zuo, Yuhao Wang, Yuan Liu and Yan Zhang, "Performance Evaluation of Routing Protocol in VANET with Vehicle-node Density", 978-1--2/10, IEEE Trans. Veh. Technol., vol. 38, no.5, pp.4244-3709 June, 2010.

[3] W. Viriysitavat, O. K.Tonguz, and F. Bai, "Network Connectivity of VANETS in Urban Areas," in Proc. IEEE SECON 2009, Rome, Italy, June 2009.

[4] Matthias Grossglauser and David N. C. Tse, "Mobility increase the capacity of wireless Ad-Hoc Networks", IEEE/ACM transactions on networking, vol. 10, no. 4, august 2002

[5] Ai HauHoYao Hua Ho, Kien A. Hua, and Roy Villafane, "A Near-Optimal Broadcast Technique for Vehicular Networks", 1-4244-2589-1/09, IEEE transactions on vehicular technology, 2009

[6] (2010) MOBISIM Tool. [Online]. Available: http://sourceforge.net/projects/mobisim/files

[7] Miguel Lu,Rodolfo Oliveira, Luis Bernardo, and Paulo Pinto, "Improving Routing Performance in High Mobility and High Density ad hoc Vehicular Networks", Universidade Nova de Lisboa, 2829-516 Caparica, Portugal uninova, 2829-516 Caparica, Portugal.

[8] (2010) NS2Website.[Online]. Available: http ns/nsbuild.html.

[9] Clausen T and P. Jacquet, "Optimized Link State Routing Protocol, IETF Internet Draft, http://www.ietf.org/internet-drafts/draft-ietf- manetolsr10.txt, 2003. 